\title{
ESTIMACIÓN DE LA EVAPOTRANSPIRACIÓN DE REFERENCIA PARA DOS ZONAS (COSTA Y REGIÓN ANDINA) DEL ECUADOR ${ }^{1}$
}

\author{
EMIL C. VEGA ${ }^{2}$, JORGE C. JARA ${ }^{3}$
}

RESUMO: Em duas zonas de latitudes similares e com diferentes altitudes, tais como Portoviejo (Região Costa) e Riobamba (Região Andina) no Equador, procurou-se ajustar os modelos de Holdridge (Ho) e Hargreaves-Samani (H-S) e com três modificações (modelos com constantes $\mathrm{C}_{\mathrm{HO}}$ e KE e KT Diário, Mensal e Anual, respectivamente) com o propósito de estimar a evapotranspiração de referência (ETo), em diferentes níveis das médias móveis do tempo, para o período de três dias. Uma vez ajustados os coeficientes $\mathrm{C}_{\mathrm{HO}}$, KE e KT, estimou-se a ETo e foram validados os resultados mediante comparação com as mensurações da ETo, utilizando-se da evaporação do tanque Classe A com seu respectivo coeficiente (ETo Pan). Nos coeficientes $\mathrm{C}_{\mathrm{HO}}$ (Ho) e KE (H-S), o melhor desempenho foi obtido pela correlação do $\mathrm{C}_{\mathrm{HO}}$ e KS com o déficit da pressão de vapor (DPV), uma vez que o mesmo corrige o gradiente térmico vertical e incrementa a qualidade da predição da ETo, enquanto correlaciona o coeficiente KT (H-S) com a temperatura do ar, permitindo corrigir a radiação solar. Na comparação das três modificações de ambos os modelos com o ETo Pan, os modelos $\mathrm{C}_{\mathrm{HO}}$ Diário e $\mathrm{H}-\mathrm{S}$ Mensal conseguiu-se as melhores estimativas da ETo com os indicadores de ajustes levemente melhores em Portoviejo e Riobamba. Finalmente, a qualidade das estimativas da ETo com três modificações de ambos os modelos é melhor em comparação com aquelas do modelo da Penman-Monteith para ambas as localidades.

PALAVRAS-CHAVE: evapotranspiração de referência (ETo), altitude, temperatura, déficit de pressão de vapor, Hargreaves-Samani.

\section{ESTIMATION OF CROP REFERENCE EVAPOTRANSPIRATION FOR TWO LOCATIONS (COASTAL AND ANDEAN REGION) OF ECUADOR}

\begin{abstract}
In two locations of Ecuador with similar latitude but different altitude (Portoviejo, Coastal zone and Riobamba, Andean zone) the Holdrigde (Ho) and Hargreaves-Samani (H-S) models and three modifications of these (daily, monthly and annual with constants $\mathrm{C}_{\mathrm{HO}}$ and $\mathrm{KE}$ and $\mathrm{KT}$, respectively) were fitted in order to estimate crop reference evapotranspiration (ETo) for a range of moving averages in time, for a three day period. Once $\mathrm{C}_{\mathrm{HO}}, \mathrm{KE}$ and $\mathrm{KT}$ were calibrated those ETo estimates were compared with ETo estimated by the Pan Evaporation Method (ETo Pan). Among $\mathrm{C}_{\mathrm{HO}}(\mathrm{Ho})$ and $\mathrm{KE}(\mathrm{H}-\mathrm{S})$ coefficients, the best performance was achieved relating $\mathrm{C}_{\mathrm{HO}}$ and $\mathrm{KE}$ to vapor pressure deficit, since it corrected the vertical thermal gradient and increased the precision in ETo prediction, while relating the KT $(\mathrm{H}-\mathrm{S})$ coefficient to air temperature allowed the correction of the solar radiation. When comparing the three modifications of both models with ETo Pan, the daily $\mathrm{C}_{\mathrm{HO}}$ model and the monthly $\mathrm{H}-\mathrm{S}$ model generated slightly better ETo estimates in Portoviejo and Riobamba, respectively. Finally, in terms of quality of ETo estimates, any of the three modifications of both models were better than those with Penman-Monteith in both locations.
\end{abstract}

KEYWORDS: reference evapotranspiration, altitude, temperature, vapor pressure deficit, Hargreaves-Samani.

\footnotetext{
${ }^{1}$ Parte de la Tesis para optar el grado de Magíster en Ingeniería Agrícola - mención Recursos Hídricos, presentada por el primer autor a la Facultad de Ingeniería Agrícola de la Universidad de Concepción, Chile.

${ }^{2}$ Ing. Agrícola, M.Sc., Departamento de Recursos Hídricos, Facultad de Ingeniería Agrícola, Universidad de Concepción, Chillán Chile.

${ }^{3}$ Ing. Agronomo, M.Sc. Ph.D, Profesor Asociado, Departamento de Recursos Hídricos, FIA/UdeC, Chillán - Chile. Casilla de Correos 537, Fono: (56) 42 208804, e-mail: jcjara@udec.cl

Recebido pelo Conselho Editorial em: 11-4-2007

Aprovado pelo Conselho Editorial em: 12-8-2009
}

Eng. Agríc., Jaboticabal, v.29, n.3, p.390-403, jul./set. 2009 


\section{INTRODUCCIÓN}

La Republica del Ecuador está situada al noroeste de América del Sur, posee tres regiones continentales bien definidas: la Costa, Andina y la Amazonía, que presentan diferentes condiciones climáticas determinadas por la altitud, ubicación, presencia de la Cordillera de los Andes y la influencia marítima. La Costa del Pacífico tiene una estación lluviosa entre diciembre y mayo, y otra seca desde junio a noviembre, con temperaturas medias diarias que oscilan entre los 36 y $23^{\circ} \mathrm{C}$, respectivamente. En los Andes el clima es lluvioso y frío de noviembre a abril, y seco de mayo a octubre, con temperaturas medias diarias entre $\operatorname{los} 13$ y $18{ }^{\circ} \mathrm{C}$, respectivamente. Esta gran diversidad de climas y topografía favorecen el desarrollo de diversos sistemas productivos, en donde el riego es importante dado el déficit hídrico que va desde los 1200 a los $400 \mathrm{~mm}$ anuales, desde el oeste en la costa del Pacífico hacia el este al interior del país (GOBIERNO DE LA PROVINCIA DE MANABÍ, 2004).

En el Ecuador la agricultura se desarrolla desde el nivel del mar hasta los $2800 \mathrm{~m}$ de altitud, por lo que este factor debe ser considerado en la planificación del riego. Dado que el principal problema radica en la escasa información de datos de evapotranspiración, el uso de modelos predictivos es una alternativa necesaria a desarrollar.

Considerando la amplia variedad de modelos, sobresalen aquellos que tienen una buena base física y teórica pero requieren de varios parámetros que no siempre están disponibles para su utilización, como el modelo de Penman-Monteith (P-M). Por otro lado, están aquellos más simples, que requieren sólo la temperatura del aire (Holdridge) y, adicionalmente, la latitud del lugar (Hargreaves y Samani).

El modelo de Holdridge depende linealmente de la temperatura media del aire multiplicada por un coeficiente único $\left(\mathrm{C}_{\mathrm{HO}}\right)$, mientras que el modelo de Hargreaves y Samani, además de la dependencia lineal de la temperatura media del aire, incorpora la radiación solar en función de la latitud del lugar y de la amplitud térmica del día y considera dos coeficientes: uno para obtener la radiación solar (KT) y otro para reducir los valores a evapotranspiración (KE), los cuales también pueden hacerse dependientes de la temperatura del aire. En efecto, los valores de $\mathrm{C}_{\mathrm{HO}}$ y KT generalmente son asumidos como constantes y únicos para una determinada región; sin embargo, es posible establecer una relación dinámica o de dependencia temporal con variables climáticas como temperatura (SAMANI, 2000) y humedad del aire (VEGA \& JARA, 2006), que permita incrementar la calidad de la estimación.

Modelos que involucran la temperatura del aire han sido desarrollados y/o utilizados para estimaciones de evapotranspiración en regiones tropicales y subtropicales de Ecuador, Venezuela y Brasil, en algunos casos con desempeños comparables al modelo de Penman-Monteith (MARTINEZ et al., 2005; SILVA et al., 2003; VILLACÍS, 2001; CAMARGO \& SENTELHAS, 1997). Sin embargo, es necesario considerar que para aplicar P-M rara vez se dispone de valores medidos de radiación solar y menos de radiación neta, y que el procedimiento de estimación del déficit de presión de vapor debiera ser verificado localmente (ASCE-EWRI, 2005). Por esta razón, y como procedimiento alternativo, el utilizar información de evaporación de bandeja puede proveer estimaciones aceptables de evapotranspiración de referencia dentro de los límites recomendados para este método (JENSEN et al., 1990) y, adicionalmente, permitir la comparación con otros métodos.

El objetivo de este trabajo es ajustar los modelos originales de Holdridge (Ho) y Hargreaves y Samani (H-S), para estimar evapotranspiración de referencia (ETo) con datos diarios de temperatura del aire, evaluando la variación temporal de los coeficientes de ajuste en dos lugares de similar latitud pero diferente altitud en Ecuador (Portoviejo en la Región Costa y Riobamba en la Región Andina), utilizando como elemento de validación la Eto estimada con el método de la Bandeja de Evaporación (Tanque Classe A). 


\section{MATERIAL Y MÉTODOS}

Este estudio se realizó con información meteorológica diaria de las estaciones de la Universidad Técnica de Manabí, altitud $60 \mathrm{~m}$ y coordenadas geográficas $01^{\circ} 02^{\prime} 10^{\prime \prime}$ latitud sur y 80²7'26' longitud oeste en Portoviejo (Región Costa), y de la Escuela Superior Politécnica del Chimborazo en Riobamba (Región Andina), altitud 2838 m y coordenadas geográficas 01³8'25', latitud sur y 7840'48', longitud oeste.

La estación invernal en la Región Costa (lluviosa, calurosa y húmeda) comprende los meses de diciembre a mayo y la estación de verano (seca, menos húmeda y menos calurosa) entre junio y noviembre. En la Región Andina el invierno esta comprendido desde noviembre a abril (de lluvia y frío) y la época de verano entre mayo y noviembre (seco y templado). Se utilizó información meteorológica de 1996-2005. Los datos diarios disponibles corresponden a temperatura y humedad relativa del aire (máxima, mínima y media de cinco observaciones diarias), velocidad de viento, evaporación de bandeja (excepto el 2004 en Riobamba) y horas de sol (para estimar radiación solar). Para la calibración de los modelos, se utilizó el periodo 1996-1999, mientras que para validación, el período 2000-2005.

\section{Modelos para la estimación de evapotranspiración de referencia (ETo)}

\section{Modelo de Holdridge (Ho)}

HOLDRIDGE (1959) publicó una simple expresión para estimar evapotranspiración potencial (ETp), actualmente definida como evapotranspiración de referencia (ETo) por ALLEN et al. (1998) y la ASCE-EWRI (2005), para regiones tropicales y subtropicales (excepto altas elevaciones). Esta expresión es función de la temperatura del aire comprendida entre los $0{ }^{\circ} \mathrm{C}$ y $30^{\circ} \mathrm{C}$ (biotemperatura), que determina el ritmo e intensidad de los procesos fisiológicos de las plantas (fotosíntesis, respiración y transpiración) y la tasa de evaporación directa del agua contenida en el suelo y en la vegetación. El modelo original de Holdridge tiene la siguiente forma:

$$
\mathrm{ETp}=\mathrm{C}_{\mathrm{HO}} \mathrm{T}
$$

siendo,

$$
\mathrm{C}_{\mathrm{HO}}=58,93\left(\frac{\mathrm{PUT}}{\mathrm{NUT}}\right)
$$

donde,

ETp - evapotranspiración potencial, $\mathrm{mm}_{\text {tiempo }}{ }^{-1}$;

$\mathrm{T}$ - temperatura del aire, ${ }^{\circ} \mathrm{C}$;

PUT - periodo de unidades de tiempo, días, y

NUT - número de unidades de tiempo en un año, 366 ó 365 días.

HOLDRIDGE (1959) considera $\mathrm{C}_{\mathrm{HO}}$ de 58,93 para estimaciones anuales y 0,161 para estimaciones diarias de evapotranspiración.

Una de las innovaciones importantes en el desarrollo de este modelo (TOSI, 1964) consiste en considerar la biotemperatura de manera análoga a la temperatura media del aire (diaria, mensual y/o anual); por lo tanto, para el periodo analizado el modelo de Holdridge se puede escribir como:

$$
\mathrm{ETo}=\mathrm{C}_{\mathrm{HO}} \mathrm{TP}
$$

donde,

ETo - evapotranspiración de referencia, $\mathrm{mm} \mathrm{día}^{-1}$;

$\mathrm{C}_{\mathrm{HO}}$ - coeficiente en función de alguna variable meteorológica que relacione mejor sus valores, $\mathrm{y}$

$\mathrm{TP}$ - temperatura media diaria del aire, ${ }^{\circ} \mathrm{C}$. 


\section{Modelo de Hargreaves \& Samani (H-S)}

SAMANI (2000) y HARGREAVES \& ALLEN (2003), publicaron una expresión para estimar evapotranspiración de referencia (ETo), en función de la radiación solar que llega a nivel del suelo (Rs) y el promedio de la temperatura del aire (TP), la cual está definida como:

$$
\mathrm{ETo}=\mathrm{KE} \operatorname{Rs}(\mathrm{TP}+17,8)
$$

siendo,

$$
\mathrm{Rs}=\mathrm{KT} \operatorname{Ra} \sqrt{\mathrm{TD}}
$$

donde,

ETo - evapotranspiración de referencia, $\mathrm{mm} \mathrm{día}^{-1}$;

$\mathrm{KE}$ - coeficiente para reducir los valores a ETo, $(0,0135)$;

Rs - radiación solar, $m m$ día $^{-1}$ de evaporación;

17,8 - valor que permite utilizar la temperatura del aire en grados Celsius;

$\mathrm{TP}$ - promedio de la temperatura del aire entre la máxima y mínima del día, ${ }^{\circ} \mathrm{C}$;

TD - amplitud térmica del día para períodos semanales o mensuales, ${ }^{\circ} \mathrm{C}$;

$\mathrm{Ra}$ - valor de Angot de la radiación extraterrestre al tope de la atmósfera, $\mathrm{mm}$ día ${ }^{-1}$ de evaporación, y

KT - coeficiente para reducir los valores de temperatura a radiación solar: 0,162 y 0,19 para regiones costeras e interiores, respectivamente (SAMANI, 2000).

De esta forma, al combinar la ec.(4) con la ec.(5) se simplifica el cálculo de la evapotranspiración de referencia (ETo) y queda explícitamente considerada la radiación solar, mientras que la humedad relativa implícitamente esta presente en la amplitud térmica; finalmente, el modelo queda expresado en función de temperatura y latitud (SAMANI, 2000):

$$
\mathrm{ETo}=\mathrm{KE} \mathrm{KT} \mathrm{Ra} \sqrt{\mathrm{TD}}(\mathrm{TP}+17,8)
$$

donde,

ETo - evapotranspiración de referencia, $\mathrm{mm} \mathrm{día}^{-1}$.

El coeficiente KT puede ser definido en función de la amplitud térmica si se conoce el resto de la información de la ec.(5) (SAMANI, 2000). Así mismo, KE puede ser determinado en función de alguna variable meteorológica si se cuenta con la información necesaria y si todos los valores de la ec.(6) son conocidos, excepto KE; de esta manera este coeficiente puede ser determinado mediante un proceso iterativo y su valor correlacionarlo con valores de temperatura del aire, déficit de presión de vapor, etc.

\section{Modelos para realizar el ajuste}

En este estudio se utilizaron los modelos originales de Holdridge, Hargreaves y Samani y; además, se definieron tres posibles variantes de los mismos (diaria, mensual y anual).

\section{Modelo de Holdridge}

VEGA \& JARA (2006), para la variante diaria del modelo original de Holdridge encontraron que la humedad relativa (HR) se correlacionaba con el coeficiente $\mathrm{C}_{\mathrm{HO}}$ tanto en Portoviejo como en Riobamba; sin embargo, para futuros estudios recomendaron correlacionar $\mathrm{C}_{\mathrm{HO}}$ con el déficit de presión de vapor (DPV), ya que este parámetro indica la actual demanda evaporativa del aire (ALLEN et al., 1998). Adicionalmente, en el presente estudio se utilizó integradamente la temperatura media (TP) y la amplitud térmica (TD) con la intención de mejorar los valores de $\mathrm{R}^{2}$ al correlacionarlo con $\mathrm{C}_{\mathrm{HO}}$, y dejar expresadas las variantes del modelo exclusivamente con información de temperatura del aire.

El modelo de Holdridge y sus variantes propuestas son: 
1) Modelo original de Holdridge (Ho). Donde la primera expresión del modelo es igual a 0,161 para estimaciones diarias:

$\mathrm{ETo}=0,161 \mathrm{TP}$

2) Modelo $\mathbf{C}_{\mathrm{HO}}$ Diario. Perteneciente a una variante del modelo, con $\mathrm{C}_{\mathrm{HO}}$ ya calibrado y en función del déficit de presión de vapor (DPV) o la temperatura del aire (TP y TD); por consiguiente, $\mathrm{C}_{\mathrm{HO}}$ varía según el DPV o TP y TD en forma diaria:

$$
\mathrm{ETo}=\mathrm{C}_{\text {HоD }} \mathrm{TP}
$$

3) Modelo $\mathbf{C}_{\mathbf{H O}}$ Mensual. Perteneciente a una variante del modelo, con $\mathrm{C}_{\mathrm{HO}}$ constante para cada mes que corresponda en el año, y varía según el DPV o TP y TD en forma mensual:

$$
\mathrm{ETo}=\mathrm{C}_{\text {Ном }} \mathrm{TP}
$$

4) Modelo $\mathrm{C}_{\mathrm{HO}}$ Anual. Perteneciente a una variante del modelo, con $\mathrm{C}_{\mathrm{HO}}$ constante durante todo el año:

$$
\mathrm{ETo}=\mathrm{C}_{\mathrm{HOA}} \mathrm{TP}
$$

siendo en los cuatro modelos,

ETo - evapotranspiración de referencia, $\mathrm{mm} \mathrm{día}^{-1}$, y

$\mathrm{TP}$ - temperatura media del aire de cinco observaciones diarias $(07: 00,10: 00,13: 00,16: 00 \mathrm{y}$ 19:00), ${ }^{\circ} \mathrm{C}$.

\section{Modelo de Hargreaves y Samani}

El modelo de Hargreaves y Samani y sus variantes son:

1) Modelo original de Hargreaves y Samani (H-S). Donde KE es 0,0135 y a KT le corresponden valores de 0,190 para Portoviejo $(60 \mathrm{~m})$ en la Región Costa y 0,162 para Riobamba (2838 m) en la Región Andina (SAMANI, 2000):

$$
\mathrm{ETo}=0,0135 \mathrm{KT} \operatorname{Ra} \sqrt{\mathrm{TD}}(\mathrm{TP}+17,8)
$$

2) Modelo KE-KT Diario. Perteneciente a una variante del modelo $\mathrm{H}-\mathrm{S}$, con KE y KT ya calibrados, donde KE está en función del déficit de presión de vapor (DPV) o la temperatura del aire (TP y TD), mientras que KT está únicamente en función de TP y TD; por consiguiente, KE y KT varían según el DPV o TP y TD en forma diaria:

$$
\mathrm{ETo}=\mathrm{KE} \mathrm{KT} \operatorname{Ra} \sqrt{\mathrm{TD}}(\mathrm{TP}+17,8)
$$

3) Modelo KE-KT Mensual. Perteneciente a una variante del modelo H-S, con KE y KT constantes para cada mes que corresponda en el año, según el DPV o TP y TD mensual0:

$$
\mathrm{ETo}=\mathrm{KE}_{\mathrm{M}} \mathrm{KT}_{\mathrm{M}} \mathrm{Ra} \sqrt{\mathrm{TD}}(\mathrm{TP}+17,8)
$$

4) Modelo KE-KT Anual. Perteneciente a una variante del modelo H-S, con KE y KT constantes durante todo el año:

$$
\mathrm{ETo}=\mathrm{KE}_{\mathrm{A}} \mathrm{KT}_{\mathrm{A}} \mathrm{Ra} \sqrt{\mathrm{TD}}(\mathrm{TP}+17,8)
$$

siendo en los cuatro modelos,

ETo - evapotranspiración de referencia, $\mathrm{mm} \mathrm{día}^{-1}$;

$\mathrm{Ra}$ - valor de Angot de la radiación extraterrestre al tope de la atmósfera, mm día ${ }^{-1}$ de evaporación, y

$\mathrm{TP}$ - temperatura media entre la máxima y mínima diaria, ${ }^{\circ} \mathrm{C}$. 


\section{Calibración y validación de los modelos}

La calibración y validación se realizó mediante un procedimiento similar al seguido por MERCADO \& JARA (2006):

\section{Calibración:}

- La evapotranspiración de referencia diaria (ETo Ban) se obtuvo a partir de la bandeja de evaporación multiplicada por su respectivo coeficiente de bandeja $\left(\mathrm{k}_{\mathrm{Ban}}\right)$, determinado según el procedimiento de DOORENBOS \& PRUIT (1977), el cual resultó constante para todo el año con un valor de 0,85 para Portoviejo (Región Costa) y 0,75 para Riobamba (Región Andina).

- Para la variante diaria del modelo de Holdridge, el coeficiente $\mathrm{C}_{\mathrm{HO}}$ se obtuvo en forma diaria de la ec.(3), con datos de ETo Ban y TP del periodo de calibración (1996-1999).

- Luego, los $\mathrm{C}_{\mathrm{HO}}$ diarios se correlacionaron con valores diarios de DPV para el mismo periodo de calibración, obtenido previamente por el modelo sugerido por ALLEN et al. (1998) y la ASCEEWRI (2005), y valores combinados diarios de temperatura (TP y TD), utilizando la función que obtuvo el mejor coeficiente de determinación $\left(\mathrm{R}^{2}\right)$.

- Para la variante diaria del modelo H-S, el coeficiente KT se obtuvo en forma diaria a partir de la ec.(5), con datos de radiación solar estimados previamente a partir de las horas diarias de sol con el modelo de DOORENBOS \& PRUIT (1977), y TD del periodo de calibración.

- Luego, los KT diarios se correlacionaron con datos de Rs y valores combinados diarios de temperatura (TP y TD) utilizando la función que obtenga el mejor coeficiente de determinación $\left(\mathrm{R}^{2}\right)$.

- Posteriormente, de la ec.(6), se obtiene el valor de KE (conociendo previamente Rs) para cada día y la correlación se realizó utilizando el procedimiento mencionado para el coeficiente $\mathrm{C}_{\mathrm{HO}}$.

- Para los modelos $\mathrm{C}_{\mathrm{HO}}$ Mensual (Holdrigde) y KE-KT Mensual (H-S), se obtuvieron promedios mensuales de los coeficientes $\left(\mathrm{C}_{\mathrm{HOM}}, \mathrm{KE}_{\mathrm{M}}\right.$ y $\left.\mathrm{KT}_{\mathrm{M}}\right)$ a partir de los coeficientes calibrados en forma diaria.

- Finalmente, con los modelos $\mathrm{C}_{\mathrm{HO}}$ Anual (Holdridge) y KE-KT Anual (H-S) se obtuvieron promedios anuales de los coeficientes $\left(\mathrm{C}_{\mathrm{HOA}}, \mathrm{KE}_{\mathrm{A}}\right.$ y $\left.\mathrm{KT}_{\mathrm{A}}\right)$ a partir de los coeficientes calibrados en forma diaria.

\section{Validación}

- Se estimó la evapotranspiración de referencia en forma diaria, utilizando los coeficientes $\mathrm{C}_{\mathrm{HO}}$, KE y KT calibrados en forma diaria, con promedios mensuales y promedios anuales, respectivamente. Luego de estimar ETo diaria para las cuatro variantes de ambos modelos (original, diaria, mensual y anual) en el periodo de validación (2000-2005), se calcularon promedios (medias móviles) para periodos de tres, cinco, siete, diez y treinta días (se informa sólo el periodo de tres dias) y se compararon con los periodos de igual rango de tiempo de evapotranspiración de referencia (ETo Ban) previamente estimada con la evaporación de bandeja y su respectivo coeficiente $\left(\mathrm{k}_{\mathrm{Ban}}\right)$.

- Después de validar el desempeño de cada variante del modelo, aquel que logró una mejor bondad predictiva se comparó con la evapotranspiración de referencia diaria, estimada por el modelo de Penman-Monteith (ETo P-M) (ALLEN et al., 1998), definido para un cultivo hipotético de $0,12 \mathrm{~m}$ de altura, resistencia de la cubierta vegetal de $70 \mathrm{~s} \mathrm{~m}^{-1}$ y un albedo de 0,23 :

$$
\lambda E=\frac{S\left(R_{n}-G\right)+0,0864 \rho C_{P} \frac{D P V}{r_{a}}}{S+\gamma\left(1+\frac{r_{c}}{r_{a}}\right)}
$$


donde,

$\lambda \mathrm{E}$ - flujo de calor latente, $\mathrm{MJ} \mathrm{m}^{-2}$ día $^{-1}$;

$\mathrm{S}$ - pendiente de la curva psicrométrica, $\mathrm{kPa}^{\circ} \mathrm{C}^{-1}$;

$\rho$ - densidad del aire, $\mathrm{kg} \mathrm{m}^{-3}$;

$\lambda$ - calor latente, $\mathrm{kJ} \mathrm{kg}^{-1}$;

$\gamma$ - constante psicrométrica, $\mathrm{kPa}^{\circ} \mathrm{C}^{-1}$;

$\mathrm{C}_{\mathrm{P}}$ - calor especifico del aire húmedo, $\mathrm{kJ} \mathrm{kg}^{-1}{ }^{\circ} \mathrm{C}^{-1}$;

$\mathrm{R}_{\mathrm{n}}$ - radiación neta, $M J \mathrm{~m}^{-2}$ día $^{-1}$;

$\mathrm{G}$ - flujo de calor del suelo, $\mathrm{MJ} \mathrm{m}^{-2}$ día $^{-1}$ (0 para estimaciones diarias);

$\mathrm{r}_{\mathrm{a}}$ - resistencia aerodinámica, $\mathrm{s} \mathrm{m}^{-1}$;

$\mathrm{r}_{\mathrm{c}}$ - resistencia del cultivo, $70 \mathrm{~s} \mathrm{~m}^{-1}$ (para estimaciones diarias), $\mathrm{y}$

DPV - déficit de presión de vapor, $\mathrm{kPa}$.

La determinación de la radiación neta se realizó utilizando la metodología propuesta por ALLEN et al. (1998), estimando la radiación solar a partir de información diaria de horas de sol (DOORENBOS \& PRUIT, 1977), y el déficit de presión de vapor se determino con la expresión propuesta por ALLEN et al. (1998) y la ASCE-EWRI (2005):

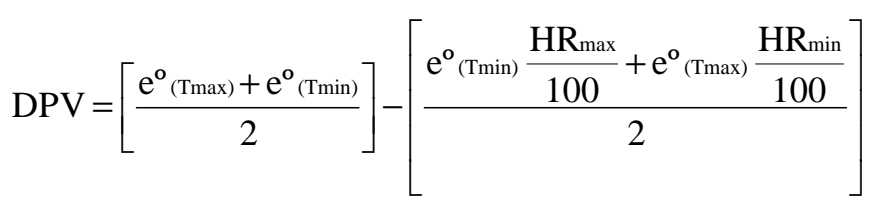

donde,

DPV - déficit de presión de vapor, $\mathrm{kPa}$;

$\mathrm{e}^{\mathrm{o}}$ - presión de vapor a saturación, $\mathrm{kPa}$, en función de la temperatura del aire;

$\mathrm{T}_{\max }$ - temperatura máxima del aire, ${ }^{\circ} \mathrm{C}$;

$\mathrm{T}_{\min }$ - temperatura mínima del aire, ${ }^{\circ} \mathrm{C}$;

$\mathrm{HR}_{\max }$ - humedad relativa máxima del aire, $\%$, y

$\mathrm{HR}_{\min }$ - humedad relativa mínima del aire, $\%$.

\section{Análisis de los datos}

Con el propósito de facilitar el contraste de la capacidad de predicción de cada modelo se utilizaron los siguientes índices estadísticos de medida de bondad de ajuste:

$$
\begin{aligned}
& \text { RMSE }=\sqrt{\frac{1}{N_{i}} \sum_{i=1}^{N}(\mathrm{Xe}-\mathrm{Xo})^{2}} \\
& \mathrm{RD}=\frac{\mathrm{RMSE}}{\overline{\mathrm{Xo}}} 100 \\
& \mathrm{MR}=\frac{1}{\mathrm{~N}_{\mathrm{i}=1}} \sum_{\mathrm{Xo}}^{\mathrm{X}} \frac{\mathrm{Xe}}{\mathrm{Xo}}
\end{aligned}
$$

donde,

RMSE - raíz del error cuadrático medio, $\mathrm{mm} \mathrm{día}^{-1}$;

$\mathrm{RD}$ - diferencia relativa, \%;

MR - cociente medio, adimensional;

Xe - valores estimados según modelo;

Xo - valores observados (ETo Ban);

$\overline{\mathrm{Xo}}$ - promedio de los valores observados, $\mathrm{y}$

$\mathrm{N}$ - número de comparaciones realizadas. 


\section{RESULTADOS Y DISCUSIÓN}

\section{Calibración de los coeficientes $\mathrm{C}_{\mathrm{HO}}$, KE y KT para los lugares de estudio}

Para ambos lugares de estudio, se realizaron 360 combinaciones entre los coeficientes $\mathrm{C}_{\mathrm{HO}}$ (Holdridge) y KE (H-S) con funciones del déficit de presión de vapor (DPV) (estimado con la ec.16), mientras que con los valores combinados de temperatura media (TP) y amplitud térmica (TD) se realizaron 1416 combinaciones. De la misma forma, con el coeficiente KT (H-S) se realizaron 1416 combinaciones con los valores combinados de temperatura media (TP) y amplitud térmica (TD).

En el Tabla 1 están representadas las mejores correlaciones obtenidas entre los coeficientes de los modelos con las funciones de las variables meteorológicas, las cuales fueron elegidas por los mejores coeficientes de determinación $\left(\mathrm{R}^{2}\right)$ logrados.

TABLA 1. Expresiones de las mejores correlaciones de los coeficientes $\mathrm{C}_{\mathrm{HO}}$ (Holdridge), $\mathrm{KE}$ y $\mathrm{KT}$ (H-S) en función de variables meteorológicas diarias. Mathematic best correlations of CHO (Holdridge), KE and KT (H-S) as a function of daily meteorological variables.

\begin{tabular}{|c|c|c|c|c|}
\hline \multirow{2}{*}{ Variables* } & \multicolumn{2}{|l|}{ Portoviejo (Región Costa) } & \multirow{2}{*}{$\begin{array}{l}\text { Riobamba (Región Andina) } \\
\text { Correlación }\end{array}$} & \multirow[b]{2}{*}{$\mathrm{R}^{2}$} \\
\hline & Correlación & $\mathrm{R}^{2}$ & & \\
\hline TP \& TD & {$\left[\operatorname{Ln}\left(\mathrm{C}_{\mathrm{HO}}\right)\right]^{-1} \mathrm{v} / \mathrm{s}\left\{\left[\operatorname{aTan}\left(\mathrm{TP}^{2}\right)\right]^{-1}[\mathrm{Ln}(\mathrm{TD})]^{-1}\right.$} & 0,11 & {$\left[\operatorname{Ln}\left(\mathrm{C}_{\mathrm{HO}}\right)\right]^{-1} \mathrm{v} / \mathrm{s}\left\{\left[\mathrm{aCos}\left(\mathrm{TD}^{0,5}\right)\right]^{-1}[\operatorname{Ln}(\mathrm{TP})]^{-1}\right\}$} & 0,13 \\
\hline $\mathrm{TP} \& \mathrm{TD}$ & $\operatorname{Ln}(\mathrm{KE}) \mathrm{v} / \mathrm{s}\left\{\left[\mathrm{aCos}\left(\mathrm{TD}^{2}\right)\right]\left(\mathrm{TP}^{-2}\right)\right\}$ & 0,04 & $\operatorname{Ln}(\mathrm{KE}) \mathrm{v} / \mathrm{s}\left\{\left[\mathrm{aSen}\left(\mathrm{TP}^{2}\right)\right] \operatorname{Ln}(\mathrm{TD})\right\}$ & 0,03 \\
\hline $\mathrm{TP} \& \mathrm{TD}$ & $\left(\mathrm{KT}^{2}\right) \mathrm{v} / \mathrm{s}\left\{\left[\mathrm{aSen}\left(\mathrm{TP}^{2}\right)\right]^{-1} \operatorname{Ln}(\mathrm{TD})\right\}$ & 0,38 & $\left(\mathrm{KT}^{2}\right) \mathrm{v} / \mathrm{s}\left\{\left[\mathrm{aSen}\left(\mathrm{TP}^{2}\right)\right]^{-1} \operatorname{Ln}(\mathrm{TD})\right\}$ & 0,37 \\
\hline DPV & {$\left[\mathrm{Ln}\left(\mathrm{C}_{\mathrm{HO}}\right)\right]^{-1} \mathrm{v} / \mathrm{s} \mathrm{DPV}{ }^{-1}$} & 0,19 & $\left(\mathrm{C}_{\mathrm{HO}}\right)^{2} \mathrm{v} / \mathrm{s} \mathrm{DPV}$ & 0,15 \\
\hline DPV & {$[\mathrm{Ln}(\mathrm{KE})]^{-1} \mathrm{v} / \mathrm{s} \mathrm{DPV}^{-1}$} & 0,14 & $\mathrm{KE}^{0,5} \mathrm{v} / \mathrm{s}[\mathrm{aCos}(\mathrm{DPV})]^{-1}$ & 0,03 \\
\hline
\end{tabular}

*TP: temperatura media diaria del aire $\left({ }^{\circ} \mathrm{C}\right.$ y/o radián); TD: amplitud térmica diaria $\left({ }^{\circ} \mathrm{C}\right.$ y/o radián); DPV: déficit de presión de vapor (kPa y/o radián); Ln: logaritmo neperiano; aCos: arco Coseno; aSen: arco Seno; aTan: arco Tangente.

En general, los valores de $\mathrm{R}^{2}$ fueron bajos e indican que los valores de los coeficientes $\mathrm{C}_{\mathrm{HO}}$, KT y KE no pueden ser explicados satisfactoriamente por las variaciones que experimentan el déficit de presión de vapor y la temperatura del aire; sin embargo, podrían contribuir a mejorar el desempeño del modelo original, especialmente en relación al uso del coeficiente KT, que reduce los valores de temperatura (TP y TD) a radiación solar. Además, se debe considerar que los coeficientes estan basados en datos meteorológicos diarios, con lo cual es esperable que los $\mathrm{R}^{2}$ sean menores a aquellos informados por SAMANI \& PESSARAKLI (1986) y SAMANI (2000), quienes utilizaron valores medios mensuales y no diarios en la determinación de KT. Finalmente, las correlaciones de los coeficientes que reducen los valores a evapotranspiración $\left(\mathrm{C}_{\mathrm{HO}} \mathrm{y} \mathrm{KE}\right)$ son mejores con el déficit de presión de vapor (DPV) que con la temperatura (TP y TD), lo que se podría explicar por la dependencia de la demanda evaporativa del aire con el DPV.

En Portoviejo (costa) la concentración de puntos se presenta entre los 0,7 y 1,4 kPa (Figura 1A y 1B Portoviejo), situación que se produce prácticamente durante todo el año entre los 23 y $28^{\circ} \mathrm{C}$ de temperatura media y amplitud térmica entre 8 y $13^{\circ} \mathrm{C}$ (Figura $1 \mathrm{C}$ Portoviejo), alcanzando valores superiores entre enero y abril, y los mínimos entre junio y agosto.

En Riobamba (Región Andina) la mayor concentración de puntos se produce entre los 0,6 y $1,2 \mathrm{kPa}$ (Figura 1A y 1B Riobamba), condiciones típicas de esta localidad durante todo el año con valores de temperatura media que fluctúan entre los 13 y $17^{\circ} \mathrm{C}$ y amplitud térmica entre 9 y $14{ }^{\circ} \mathrm{C}$ (Figura 1C Riobamba), con los mayores valores entre septiembre y noviembre y los menores entre mayo y julio.

En el caso de los promedios mensuales de los coeficientes $\mathrm{C}_{\mathrm{HOM}}$ y $\mathrm{KE}_{\mathrm{M}}$ (calibrados en función del DPV y constantes para cada mes) se puede observar (Figuras 2A y 2B) que sus valores tienden a aumentar en verano (época seca) y disminuir en invierno (época de lluvia), efecto que es más evidente en Portoviejo (Región Costa); dichos valores difieren de lo señalado por Holdridge 
$\left(\mathrm{C}_{\mathrm{HO}}-0,161\right)$ y $\mathrm{H}-\mathrm{S}(\mathrm{KE}-0,0135)$ de un valor único para el año y refuerzan la hipótesis de una dependencia temporal del valor de estos coeficientes.
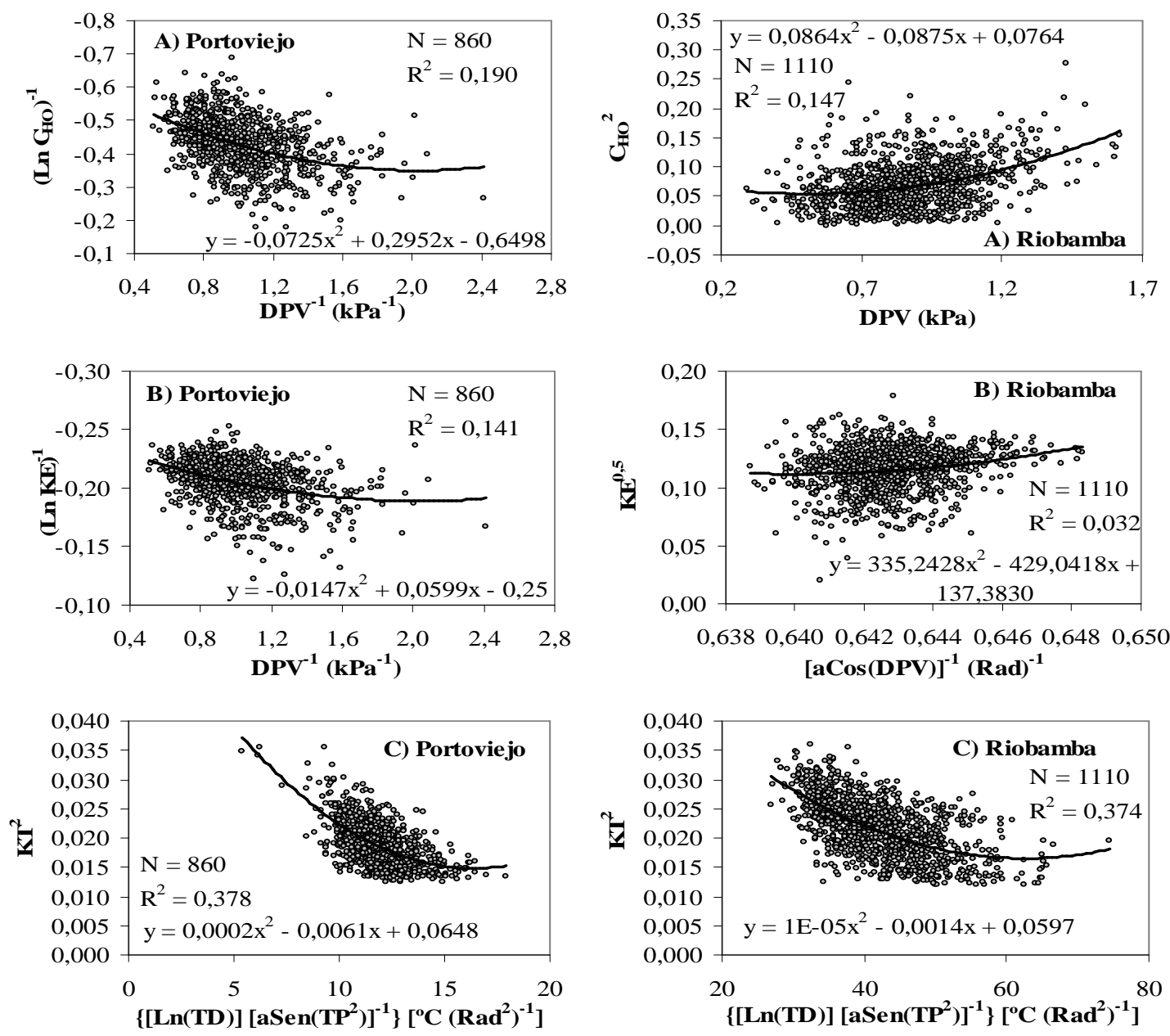

FIGURA 1. Coeficientes diarios A) $\mathrm{C}_{\mathrm{HO}}$ (Holdridge) y B) $\mathrm{KE}(\mathrm{H}-\mathrm{S})$ en función del déficit de presión de vapor diario (DPV), y C) KT (H-S) en función de la temperatura media diaria (TP) y la amplitud térmica diaria (TD) para Portoviejo (costa) y Riobamba (Región Andina). Daily coefficients A) CHO (Holdridge) and B) KE (H-S) as a function of vapor pressure deficit (DPV), and C) KT (H-S) as a function of daily average temperature (TP) and daily amplitude of the air temperature (TD) for Portoviejo (coast) and Riobamba (Andes region).

Por otro lado, en los promedios mensuales del coeficiente $\mathrm{KT}_{\mathrm{M}}$-constantes para cada mes y calibrados en función de la temperatura del aire (Figura 2C) - se aprecia que para Riobamba (Región Andina) los valores son más elevados que en Portoviejo (Región Costa), quedando demostrado que el descenso de la temperatura con la altitud se compensa en la mayoría de los casos con una radiación solar más intensa-situación típica en Ecuador (ARELLANO et al., 1993) - y refuerzan la dependencia temporal del valor de estos coeficientes.

En el TABLA 2 se observan los promedios anuales de los coeficientes $\mathrm{C}_{\mathrm{HOA}}, \mathrm{KE}_{\mathrm{A}}$ (calibrados en función del DPV) y $\mathrm{KT}_{\mathrm{A}}$ (calibrados en función de TP y TD) de los modelos de Holdridge y H$\mathrm{S}$, donde se considera un valor único para el año en la estimación de ETo. VEGA \& JARA (2006), obtuvieron valores del coeficiente $\mathrm{C}_{\mathrm{HOA}}$ (calibrado en función de la humedad relativa) de 0,0992 y 0,2657 y desviación estándar de $1,852 \times 10^{-2}$ y $2,798 \times 10^{-2}$ para Portoviejo y Riobamba, respectivamente. El valor mayor que presenta Riobamba (Región Andina) podría indicar una zona menos húmeda que Portoviejo (costa), y que la temperatura media durante todo el año en dos 
lugares de similar latitud y diferente altitud es menor a mayores altitudes, considerando que el gradiente térmico vertical para el caso particular de Ecuador es de $4,5^{\circ} \mathrm{C}$ de disminución por cada kilómetro de altura (PÉREZ, 1997).

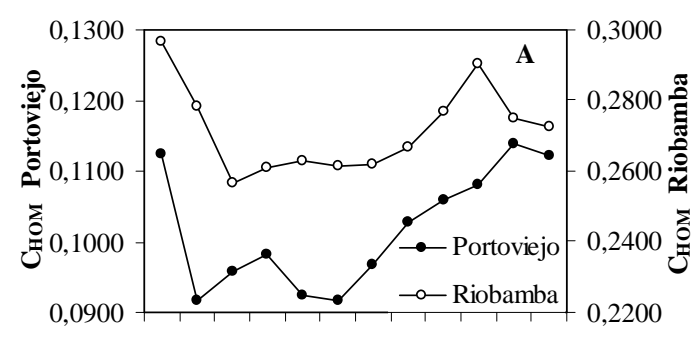

E F M A M Jn J A S O N D Meses

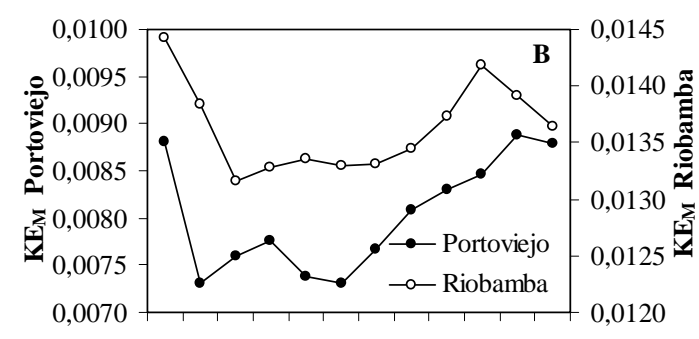

E F M A M Jn J A S O N D Meses

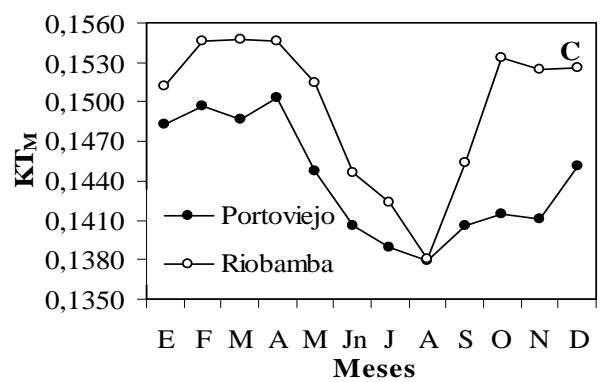

FIGURA 2. Coeficientes mensuales $\mathrm{C}_{\mathrm{HO}}$ y $\mathrm{KE}$ (calibrados en función del déficit de presión de vapor) y KT (calibrado en función de la temperatura media y amplitud térmica) de los modelos modificados de Holdridge y $\mathrm{H}-\mathrm{S}\left[\mathrm{C}_{\mathrm{HOM}}(\mathrm{A}), \mathrm{KE}_{\mathrm{M}}(\mathrm{B})\right.$ y $\left.\mathrm{KT}_{\mathrm{M}}(\mathrm{C})\right]$ para Portoviejo (costa) y Riobamba (Región Andina). $\mathbf{C}_{\mathbf{H O}}$ and $\mathrm{KE}$ monthly coefficients (calibrated as a function of vapor pressure deficit) and KT (calibrated as a function of average temperature and amplitude of the air temperature) of Holdridge and $\mathrm{H}-\mathrm{S}$ modified models $\left[\mathrm{C}_{\mathrm{HOM}}(\mathrm{A}), \mathrm{KE}_{\mathrm{M}}(\mathrm{B})\right.$ and $\left.\mathrm{KT}_{\mathrm{M}}(\mathrm{C})\right]$ for Portoviejo (coast) and Riobamba (Andes region).

RIVANO \& JARA (2006) informaron de una zona húmeda del sur de Chile, donde el valor obtenido de KE resultó menor a lo informado por SAMANI \& PESSARAKLI (1986), SAMANI (2000) y MERCADO (2006). De la misma forma, se aprecia que en Portoviejo (TABLA 2) el valor del coeficiente anual $\mathrm{KE}_{\mathrm{A}}$ es menor en un $41 \%$ al obtenido en Riobamba, teniendo este ultimo una mayor aproximación al valor de 0,0135 del modelo $\mathrm{H}-\mathrm{S}$ original; así mismo, el coeficiente $\mathrm{KT}_{\mathrm{A}}$ con un valor de 0,1499 obtenido en Riobamba- es mayor (por el efecto de la altitud) al obtenido en Portoviejo.

TABLA 2. Promedios anuales de los coeficientes $\mathrm{C}_{\mathrm{HOA}}, \mathrm{KE}_{\mathrm{A}}$ (calibrados en función del déficit de presión de vapor) y $\mathrm{KT}_{\mathrm{A}}$ (calibrados en función de la temperatura media y amplitud térmica) de los modelos $\mathrm{C}_{\mathrm{HO}}$ Anual (Holdridge) y KE-KT Anual (H-S). Annual averages of CHOA, KEA (calibrated as a function of vapor pressure deficit) and KTA (calibrated as a function of average temperature and amplitude of the air temperature) coefficients of CHO Annual (Holdridge) and KE-KT Annual (H-S) models.

\begin{tabular}{lcccrl}
\hline Coeficiente Anual & $\begin{array}{c}\text { Portoviejo } \\
\text { (Región Costa) }\end{array}$ & SD & $\begin{array}{c}\text { Riobamba } \\
\text { (Región Andina) }\end{array}$ & \multicolumn{1}{c}{$\mathrm{SD}$} \\
\hline $\mathrm{C}_{\mathrm{HOA}}$ & 0,1029 & 1,90 & $10^{-2}$ & 0,2734 & $3,4910^{-2}$ \\
$\mathrm{KE}_{\mathrm{A}}$ & 0,0081 & 1,35 & $10^{-3}$ & 0,0137 & $1,1410^{-3}$ \\
$\mathrm{KT}_{\mathrm{A}}$ & 0,1442 & 8,97 & $10^{-3}$ & 0,1499 & $11,8010^{-3}$ \\
\hline
\end{tabular}




\section{Validación de los modelos}

El estudio se concentró en el rango de promedios móviles de tres días; sin embargo, se observó que a medida que aumentaba el número de días incluidos en las medias móviles, la calidad de la predicción de ETo mejoraba significativamente (VEGA, 2007).

En general, las variantes propuestas a los modelos de Holdridge y H-S permiten mejorar la calidad de estimación de ETo en relación a los modelos originales, y todos ellos muestran un mejor desempeño en la costa. En Portoviejo (costa), a igualdad de periodo de tiempo considerado, la mejor capacidad de predicción la presentaron las variantes del modelo de Holdridge por sobre las variantes de H-S, con los menores valores de RMSE y RD (Cuadro 3), situación que puede ser explicada por las condiciones climáticas tropicales que durante todo el año presenta esta localidad, además de su baja altitud en relación al nivel del mar $(60 \mathrm{~m})$. Sin embargo, dichas diferencias no son relevantes, siendo las variantes diarias de ambos modelos las de mejor desempeño en la costa.

En contraste, en Riobamba (Región Andina) los indicadores estadísticos muestran un mejor desempeño en todas las variantes del modelo de H-S por sobre el modelo de Holdridge (Cuadro 3), lo que puede ser explicado por la limitación de uso de este ultimo modelo en zonas altas. Así mismo, es importante destacar que en Riobamba la variantes mensual de H-S logró los mejores indicadores de ajustes.

TABLA 3. Indicadores de ajuste de ETo para el modelo de Holdridge $(\mathrm{Ho})$ y sus variantes: $\mathrm{C}_{\mathrm{HO}}$ Diario, Mensual y Anual; Hargreaves y Samani (H-S) y sus variantes: KE-KT Diario, Mensual y Anual; y Penman-Monteith (P-M), comparados con ETo Ban, para rangos de promedios móviles de tiempo de tres días, periodo 2000-2005. ETo goodness of fit statistical indicators for the Holdridge (Ho) model and their variants: CHO daily, monthly and annual; Hargreaves y Samani (H-S) and their variants: KE-KT daily, monthly and annual; and Penman-Monteith (P-M), compared with ETo Pan, for a range of three days mobile averages in time, 2000-2005 period.

\begin{tabular}{|c|c|c|c|c|c|c|c|c|c|c|}
\hline \multirow{3}{*}{$\begin{array}{c}\text { Indicadores } \\
\text { de Ajuste }\end{array}$} & \multirow{3}{*}{ Localidad } & \multicolumn{9}{|c|}{ Modelos } \\
\hline & & \multirow{2}{*}{ Ho } & \multicolumn{3}{|c|}{$\mathrm{C}_{\mathrm{HO}}$} & \multirow{2}{*}{$\mathrm{H}-\mathrm{S}$} & \multicolumn{3}{|c|}{ KE-KT } & \multirow{2}{*}{$\mathrm{P}-\mathrm{M}$} \\
\hline & & & Diario & Mensual & Anual & & Diario & Mensual & Anual & \\
\hline RMSE & Portoviejo & 1,25 & 0,69 & 0,77 & 0,80 & 2,80 & 0,72 & 0,79 & 0,81 & 1,16 \\
\hline RD & (Región & 41,73 & 23,16 & 25,84 & 26,87 & 93,65 & 24,19 & 26,28 & 27,11 & 38,76 \\
\hline MR & Costa) & 1,45 & 0,91 & 0,91 & 0,92 & 2,03 & 0,91 & 0,91 & 0,93 & 1,41 \\
\hline RMSE & Riobamba & 1,27 & 1,15 & 1,09 & 1,12 & 1,13 & 0,98 & 0,95 & 0,95 & 1,21 \\
\hline RD & Región & 38,84 & 35,14 & 33,24 & 34,07 & 34,36 & 30,02 & 28,92 & 28,99 & 36,97 \\
\hline MR & Andina & 0,80 & 1,35 & 1,35 & 1,36 & 1,36 & 1,25 & 1,25 & 1,26 & 1,41 \\
\hline
\end{tabular}

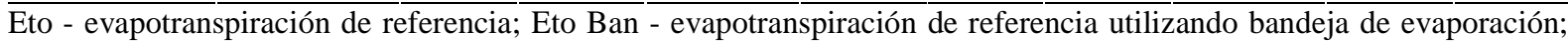
RMSE - raíz del error cuadrático medio $\left(\mathrm{mm} \mathrm{día}^{-1}\right)$; RD - diferencia relativa (\%); MR - cociente medio.

\section{Comparación entre los modelos $\mathrm{C}_{\mathrm{HO}}$ Diario y KE-KT Diario con el modelo Penman-Monteith}

En Portoviejo (costa), el valor de RMSE fue inferior a $1 \mathrm{~mm} \mathrm{día}^{-1}$ tanto en el modelo $\mathrm{C}_{\mathrm{HO}}$ Diario (Holdridge) como en KE-KT Diario (H-S), mientras que en el modelo de Penman-Monteith este indicador fue superior a $1 \mathrm{~mm} \mathrm{día}^{-1}$ y su valor de RD supera el $38 \%$ (Figura 3). El modelo de Holdridge $\mathrm{C}_{\mathrm{HO}}$ Diario es altamente recomendable para Portoviejo (costa) destacándose, además de la mínima información que requiere el modelo, la tendencia a seguir mejor la recta 1:1, tal como lo refleja el valor de su pendiente y el intercepto en la ordenada (Figura 3A Portoviejo). Asimismo, la variante KE-KT Diario del modelo de H-S también puede ser considerada para estimar ETo en la costa (Figura 3B).

En lo concerniente a Riobamba (Región Andina), los valores de RMSE, RD y MR son mayores a los obtenidos en Portoviejo para las tres variantes de ambos modelos; sin embargo, las diferencias producidas entre estos modelos con el de P-M fueron menores que los obtenidos en la costa (TABLA 3). Así mismo, hay una sobrestimación de ETo bajo los 4,5 mm día ${ }^{-1}$, 
principalmente $\mathrm{C}_{\mathrm{HO}}$ Diario y P-M (Figura 3A y 3C), con los mayores valores de RMSE, RD y MR en P-M. Aún cuando la variante KE-KT Mensual del modelo de H-S obtuvo el mejor desempeño, con los indicadores estadísticos levemente de mejor calidad que el modelo KE-KT Diario, las tres variantes propuestas del modelo de H-S (diaria, mensual y anual) también pueden recomendarse para Riobamba.

La baja calidad de predicción de P-M, dada por los valores elevados de RMSE, RD y MR, son producto de una considerable sobrestimación de ETo, que se produce prácticamente a lo largo de toda la recta 1:1 (Figura 3C) y podría ser explicada por la metodología utilizada para estimar el déficit de presión de vapor (ec. 16) y/o la estimación de la radiación neta. Además, la estación meteorológica en esta localidad se encuentra dentro de un jardín botánico, induciendo altos valores de humedad relativa; así mismo, la presencia de árboles al actuar como barreras reductoras en este lugar puede ser responsable de las bajas velocidades de viento registradas en la estación que, en general, no superaron los $2 \mathrm{~m} \mathrm{~s}^{-1}$.
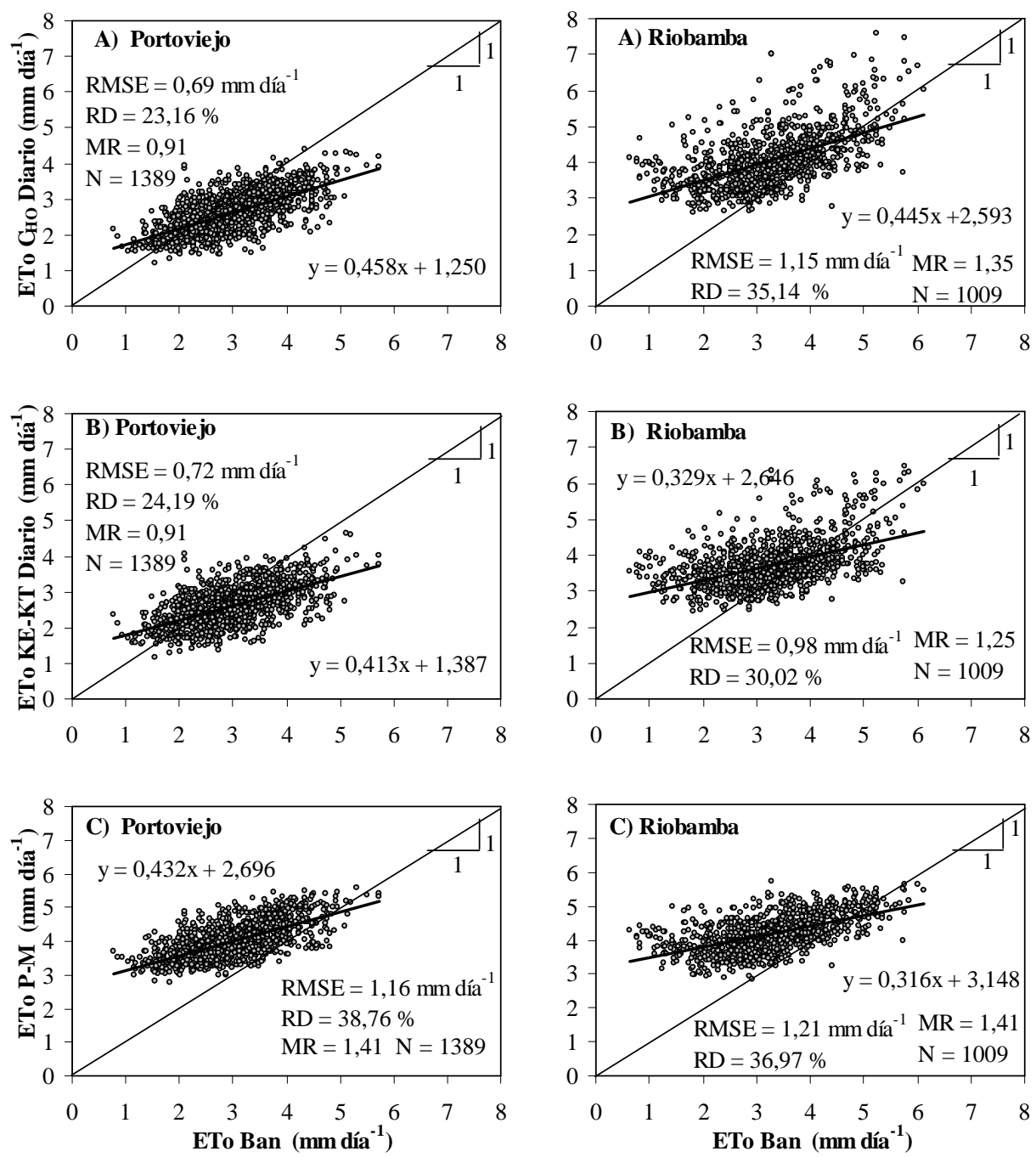

FIGURA 3. Evapotranspiración de referencia (ETo) estimada para un periodo móvil de tres días, obtenida con los modelos de: A) $\mathrm{C}_{\mathrm{HO}}$ Diario (Holdridge), B) KE-KT Diario (H-S) y C) Penman-Monteith (P-M) en una representación 1:1, comparada con ETo Ban durante el periodo 2000-2005 para Portoviejo (costa) y Riobamba (Región Andina). Reference evapotranspiration (ETo) estimated for a mobile period of three days, using the models: A) C CHO daily (Holdridge), B) KE-KT daily (H-S) and C) PenmanMonteith (P-M) in a 1:1 representation, compared with ETo Pan during the 2000-2005 period for Portoviejo (coast) and Riobamba (Andes region). 


\section{CONCLUSIONES}

$\mathrm{Al}$ evaluar las variantes propuestas de Holdridge y Hargreaves y Samani, el modelos $\mathrm{C}_{\mathrm{HO}}$ Diario seguido del modelo KE-KT Diario, lograron las mejores estimaciones de evapotranspiración de referencia en Portoviejo (Región Costa)- lo que indicaría para esta localidad una cierta dependencia temporal de los coeficientes de ambos modelos-, mientras que las tres variantes propuestas del modelo de Hargreaves y Samani (diaria, mensual y anual) tuvieron mejor desempeño en Riobamba (Región Andina), siendo levemente superior el comportamiento de la variante mensual.

Al comparar las tres variantes propuestas de ambos modelos, se observó que la calidad de las estimaciones fueron mejores que las obtenidas con P-M en ambas localidades, destacándose finalmente la variante del modelo de Holdridge por su extrema simplicidad y el mínimo requerimiento de parámetros meteorológicos.

\section{AGRADECIMIENTOS}

Los autores agradecen al Instituto Nacional de Meteorología e Hidrología del Ecuador, a la Universidad Técnica de Manabí y la Escuela Superior Politécnica del Chimborazo, por las facilidades otorgadas para la recopilación de la información meteorológica.

\section{REFERENCIAS}

ALLEN, R.; PEREIRA, L.S.; RAES, D.; SMITH, M. Guidelines for computing crop water requirements. Rome: FAO, 1998. 310 p.(Irrigation and Drainage Paper, 56).

ARELlANO, R.; CARVAJAL, G.; MOYA, R.; CARVAJAL, M. Cálculo de la evapotranspiración potencial por varios métodos para cien localidades ecuatorianas. Quito: Instituto Nacional de Meteorología e Hidrología, 1993. 100 p.

ASCE-EWRI. The ASCE standardized referent crop evapotranspiration equation. Reston:

Technical Committee Report on Environmental and Water Resource Institute of the American Society of Civil Engineers from the Task Committee on Standardization of Reference Evapotranspiration, 2005. 70 p.

CAMARGO, Â.; SENTELHAS, P. Avaliação do desempenho de diferentes métodos de estimativa da evapotranspiração potencial no Estado de São Paulo, Brasil. Revista Brasileira de Agrometeorologia, Santa Maria, v.5, n.1, p.89-97, 1997.

DOORENBOS, J.; PRUITT W.O. Guidelines for predicting crop water requirements. Rome: FAO, 1977. 179 p. (Irrigation and Drainage Paper, 24).

GOBIERNO DE LA PROVINCIA DE MANABÍ. Mapas de cantones expuestos a sequías y déficit hídrico. Plan de desarrollo provincial. Protoviejo: Secretaría de Planificación GPM, 2004.

HARGREAVES, G.H.; ALLEN, R.G. History and evaluation of Hargreaves evapotranspiration equation. Journal of Irrigation and Drainage Engineering, Reston, v.129, n. 1, p.53-63, 2003.

HOLDRIDGE, L.R. Simple method for determining potential evapotranspiration from temperature data. Science, Washington, v.130, n.3375, p.572, 1959.

JENSEN, M.E.; BURMAN, R.D.; ALLEN, R.G. (Ed.). Evapotranspiration and irrigation water requirements., New York: American Society of Civil Engineers, 1990. 332 p. (ASCE Manuals and Report on Engineering Practice, 70)

MARTINEZ, R.; BOUERI, M.; ESCALONA, P. Correlación entre la evaporación en tina y la evapotranspiración de referencia en cinco estaciones climatológicas de Venezuela. Bioagro, Barquisimeto, v.17, n.2, p.79-83, 2005. 
MERCADO, G.H. Estimación de la evapotranspiración de referencia utilizando información de temperatura del aire. 2006. 123 f. Tesis (Magíster Ingeniería Agrícola) - Departamento de Recursos Hídricos, Facultad de Ingeniería Agrícola, Universidad de Concepción, Chillán, 2006.

MERCADO, G.H.; JARA, J.C. Estimación de la evapotranspiración de referencia utilizando información de temperatura del aire. In: CONGRESO LATINOAMERICANO Y DEL CARIBE DE INGENIERÍA AGRÍCOLA, 7., CONGRESO INTERNACIONAL DE INGENIERÍA AGRÍCOLA, 5., 2006, Chillán. Actas... Chillán: Universidad de Concepción, 2006. 1 CD-ROM.

PÉREZ, S.A. Cálculo de la necesidad de agua de las plantas con datos meteorológicos. 2.ed. Riobamba: Freire, 1997. 289 p.

RIVANO, F.M.; JARA, J.C. Estimación de la evapotranspiración de referencia en la localidad de Remehue-Osorno, X Región, Chile. In: CONGRESO IBEROAMERICANO DE RIEGO Y DRENAJE, 6., 2006, Lima. Actas... Lima: Universidad Nacional Agraria La Molina, 2006. 1 CD-ROM.

SAMANI, Z.A. Estimating solar radiation and evapotranspiration using minimum climatological data. Journal of Irrigation and Drainage Engineering, Reston, v.126, n.4, p.265-267, 2000.

SAMANI, Z.A.; PESSARAKLI, M. Estimating potential crop evapotranspiration with minimum data in Arizona. Transactions of the ASAE, St. Joseph, v.29, n.2, p.522-524, 1986.

SILVA, T.J.; MONTENEGRO, A.A.; RODRÍGUEZ, J.J.; BONFIM, E. Aplicação de lisímetro de pesagem hidráulica na determinação da evapotranspiração de referência, em Petrolina - PE, Brasil. Engenharia Agrícola, Jaboticabal, v.23, n.3, p.511-520, 2003.

TOSI, J.A. Climatic control of terrestrial ecosystems: A report on the Holdridge model. Economic Geography, Worcester, v.40, n.2, p.173-181, 1964.

VEGA, E. Estimación de la evapotranspiración de referencia para dos zonas (costa y región andina) del Ecuador. 2007. 100 f. Tesis (Magíster Ingeniería Agrícola) - Departamento de Recursos Hídricos, Facultad de Ingeniería Agrícola, Universidad de Concepción, Chillán, 2007.

VEGA, E.C.; JARA, J.C. Estimación de la evapotranspiración de referencia utilizando la temperatura del aire en dos regiones del Ecuador. In: CONGRESO NACIONAL DE INVESTIGACIÓN, TECNOLOGÍA E INNOVACIÓN, 11., 2006, Guayaquil. Actas... Guayaquil: Escuela Superior Politécnica del Litoral, 2006. 1 CD-ROM.

VILLACÍS, F. Estudio meteorológico de la Provincia de Manabí. 2001. 110 f. Tesis (Ingeniero Agrónomo) - Facultad de Ingeniería Agronómica, Universidad Técnica de Manabí, Portoviejo, 2001. 